\title{
Enterocutaneous Fistulas: A Look at Causes and Management
}

\author{
Carla I. Haack · John R. Galloway • \\ Jahnavi Srinivasan
}

Published online: 16 September 2014

(C) Springer Science + Business Media New York 2014

\begin{abstract}
Despite advances in medical technology and surgical care, the management of enterocutaneous fistulas remains one of the most challenging problems faced by physicians. Success depends on an expert multidisciplinary team, access to long-term enteral and parenteral nutrition support, advanced wound care, optimal medical management and meticulous, methodical, surgical decision-making and technique. Management is complex and multiphasic. Improved survival rates for many morbid problems have resulted in a growing population of patients with increasingly complex fistulas. This article reviews the etiologies as well as classic and evolving management strategies for this problem.
\end{abstract}

Keywords Enterocutaneous fistula - Etiology ·

Mortality $\cdot$ Surgery $\cdot$ Nutritional support $\cdot$ Wound care

\section{Introduction}

The eruption of an enterocutaneous fistula (ECF) is one of the most troubling and disheartening experiences patients and their physicians will encounter. Despite advances in nutrition support, antibiotics, and surgical critical care, the

This article is part of the Topical Collection on Acute Care Surgery.

C. I. Haack · J. R. Galloway $(\bowtie) \cdot$ J. Srinivasan

Emory University Hospital, 1364 Clifton Rd NE, Atlanta,

GA 30322, USA

e-mail: jgall02@emory.edu

C. I. Haack

e-mail: chaack@emory.edu

J. Srinivasan

e-mail: jsrini2@emory.edu management of enterocutaneous fistulas remains one of the most challenging surgical problems surgeons face today. Even in the most experienced hands and specialized centers, mortality remains $5-15 \%[1,2,3 \cdot, 4-7]$. Successful closure, either by operative or non-operative means, involves a fine balance of vigilance and anticipation, optimal medical management, meticulous surgical technique, and sound decision making along with social and emotional support for the patient and their family. A wellorganized management strategy utilizing of a multidisciplinary team approach is essential. A remarkable amount of time and labor along with intensive resource utilization yields closure rates ranging from 5 to $20 \%$ without surgical intervention and 75-85\% with definitive operative treatment $[2,3 \cdot, 4,57-11]$. The team members involved in the care of these complex patients include: general and reconstructive surgeons, nutritional support staff, bedside and enterostomal nursing, social workers, radiologists, internists, psychiatrists and physical therapists, among others [1]. While mortality rates have improved over the past four decades, leading institutions with dedicated surgeons and full multidisciplinary teams well versed in the management of these patients continue to publish very high morbidity rates, in excess of $85 \%[3 \cdot, 12]$.

The initial morbidity and mortality of an enterocutaneous fistula stems from the classic triad are described by Edmunds et al. as sepsis, malnutrition and fluid/electrolyte abnormalities [3•, 9, 13]. Once the fistula has declared itself, the four principles outlined by Chapman et al. [14] in 1964 regarding the initial care of patients with ECF still hold true: (1) correction of intravascular volume, (2) drainage of abscess, (3) control of output, and (4) protection of skin.

Strict adherence to the following principles of management is paramount: (1) identification of the fistula; (2) 
Table 1 Spontaneous closure based on anatomy or site of origin

\begin{tabular}{ll}
\hline Favorable & Unfavorable \\
\hline Esophagus & Stomach \\
Duodenal Stump & Duodenum \\
Pancreas & Proximal jejunum \\
Biliary tree & Ileum \\
Colon & \\
\hline
\end{tabular}

resuscitation and sepsis control with correction of electrolyte imbalances; (3) protection of the skin and control of fistula output; (4) nutritional support; (5) radiographic investigation; and (6) definitive management, potentially with operative repair [11]. Long-term morbidity and mortality are associated with recurring sepsis, failed operative closure with recurrent ECF, short bowel syndrome, and cholestatic liver failure.

The ultimate goal in managing enterocutaneous fistulas is restoration of gastrointestinal continuity while minimizing morbidity and mortality.

\section{Classification}

Fistulas can be classified by their anatomy, physiology, or etiology. All of these factors contribute to the morbidity, mortality, and likelihood of spontaneous closure [11].

The anatomy of a fistula will depend on the organs involved, the presence or absence of associated abscess cavities, and the length and characteristics of the fistula tract. Fistulas arising from the esophagus, duodenal stump after gastric resection, pancreaticobiliary tract, and jejunum are more likely to close without operative intervention. Additionally, those with long tracts and small enteric wall defects are associated with higher spontaneous closure rates [15]. In contrast, those associated with the stomach, the lateral wall of the duodenum, the ligament of Treitz, and the ileum are more likely to require surgical correction (Table 1). Other anatomical factors that decrease the likelihood of nonoperative closure include complete disruption of GI continuity, adjacent abscess, strictured or diseased bowel, foreign bodies or distal intestinal obstruction [15] (Table 2).

The physiology of the fistula will be determined by the volume and character of its output. High output fistulas are those which drain more than $500 \mathrm{ml} /$ day, moderate output drain $200-500 \mathrm{ml} /$ day, and low output drain less than $200 \mathrm{ml} /$ day $[15,11]$. Careful monitoring of the character and volume of output of a fistula can guide resuscitation and nutrition support efforts. There have been several series studying the relationships between output and mortality as well as likelihood of spontaneous closure suggesting increased mortality and decreased spontaneous
Table 2 Spontaneous closure based on fistula character and environment

\begin{tabular}{lll}
\hline & Favorable & Unfavorable \\
\hline Enteric defect & $<1 \mathrm{~cm}$ & $>1 \mathrm{~cm}$ \\
Fistula tract & $>3 \mathrm{~cm}$ & $<3 \mathrm{~cm}$ \\
Budding mucosa & Absent & Present \\
Intestinal continuity & Intact & Disrupted \\
Distal obstruction & Absent & Present \\
Adjacent abscess & Absent & Present \\
Disease bowel & Absent & Present \\
Foreign body & Absent & Present \\
Previous radiation & No & Yes \\
\hline
\end{tabular}

Table 3 Spontaneous closure based on physiology

\begin{tabular}{lll}
\hline & Favorable & Unfavorable \\
\hline Fistula output & Low-med & High \\
& $(<500 \mathrm{cc} / \mathrm{d})$ & $(>500 \mathrm{cc} / \mathrm{d})$ \\
Nutrition status & Well-nourished & Malnourished \\
Transferrin & $>200 \mathrm{mg} / \mathrm{dl}$ & $<200 \mathrm{mg} / \mathrm{dl}$ \\
Sepsis & Absent or infrequent & Present or frequent \\
\hline
\end{tabular}

closure rate in high output fistulas [13, 16-19]. Other physiological factors that predict spontaneous fistula closure include a well-maintained nutritional status and absence of sepsis (Table 3). One study suggests that a low C-reactive protein-to-albumin ratio, which measures the relationship between acute inflammation and nutrition, is a useful predictor of spontaneous fistula closure [20].

The etiology of the fistula also bears on the likelihood of non-operative closure. The formation of a fistula generally occurs by intestinal disease extending to surrounding structures, normal bowel becoming involved in extraintestinal pathology, trauma or anastomotic breakdown [11].

Approximately $75-85 \%$ of fistulas occur following operative intervention [11, 19]. Commonly associated procedures are those carried out for malignancy, inflammatory bowel disease (IBD), adhesions, pancreatitis or abdominal wall hernia repair. Causes include breakdown of an anastomotic suture line, unintentional enterotomy or inadvertent bowel injury at the time of closure [21, 22]. Recent series show that the incidence of ECF related to mesh implantation for hernia repair is on the rise [3•]. Mesh can be responsible for formation of a fistula regardless of the type of mesh or technique used in placement. Fistulas tend to occur at the periphery of the mesh where shear forces between the mesh and the underlying bowel are greatest. The authors have also seen fistulas occur due to adherence of the intestines to metal tacks used in securing the mesh and subsequent erosion into bowel. 
During the past two decades, wide adoption of damage control laparotomy techniques has brought about an increasing number of complex fistulas [23]. These so called enteroatmospheric fistulas (EAF) arise in the setting of an open wound without abdominal musculature or skin surrounding a budded or "stomatized" fistula opening in the midst of a large bed of granulation tissue [23-25]. Management of such complex wounds requires novel ways of controlling fistula output and protecting skin. Finally, patients with multiple prior surgeries resulting in the need for extensive adhesiolysis along with emergency operations often performed in the settings of hypotension, hypothermia, anemia, and poor tissue oxygen delivery increase the risk of fistula formation. In light of the fact that the majority of fistulas are iatrogenic, the best treatment has always been and remains prevention by means of sound surgical technique and medical practice. This includes adequate preoperative patient preparation, meticulous intraoperative technique, and detailed, diligent post-operative care.

15-20\% of ECF arise spontaneously in the setting of underlying pathology. Common causes include IBD, radiation enteritis, diverticular disease, appendicitis, perforated ulcers, malignancy, intra-abdominal sepsis, pancreatitis, ischemia, and trauma. Ability to correct or treat the underlying cause bears heavily on the non-operative closure rate $[18,26]$.

\section{Management}

Successful management of the ECF presents complex physiological, mechanical, and anatomical challenges. It is often a long-term, multi-phase process [7]. A successful outcome with closure rates up to $85 \%$ requires a multidisciplinary approach utilizing the knowledge and skills of not only the surgeon, but also wound and ostomy nurses, a specialized nutritional support team, intensive care and general floor nursing, physical and occupational therapy, medical social workers, pain management, psychiatry, palliative care and spiritual support services.

\section{Identification}

The diagnosis of a fistula generally relates to recognition of enteric contents draining from a wound. This can be confirmed by the use of radiographic studies showing extravasation of enteral contrast. That said, a high index of suspicion for intestinal compromise in a patient who has undergone an abdominal operation with a sluggish "off trajectory" early post-operative course can lead to earlier detection and intervention before sepsis and organ failure occur.

\section{Resuscitation}

The goal of this phase of care is restoration of intravascular volume and optimization of oxygen carrying capacity [11]. The primary aim should be to replace the volume lost externally through the fistula or the open abdomen, as well as internally via third spacing. Caution should be exercised to avoid over-resuscitation which can lead to Abdominal compartment syndrome as well as anastomotic complications. Placement of a urinary catheter to monitor urinary output closely as a guide to adequate fluid replacement is essential. Transfer to the ICU and placement of a central venous catheter may be required for hemodynamic monitoring, especially when sepsis is suspected. Vigorous replacement of electrolytes and judicious blood transfusion are often necessary.

\section{Control of Sepsis}

Sepsis is a major source of morbidity and mortality in this patient population. Therefore, its management should be prioritized and occur concomitantly with resuscitation. CT scan or ultrasound can reveal intra-abdominal abscesses requiring drainage [2, 3•, 4-9]. Drains can be placed and cultures obtained percutaneously under image guidance or operatively. In situations with significant ongoing spillage or incomplete abscess decompression, operative "tractotomy" along existing drains with placement of larger irrigating drains or open surgical drainage with or without proximal diversion can be considered. Attempts at definitive repair should be avoided at this time. Once an abscess has been decompressed, the drains should be injected with water-soluble contrast to delineate the anatomy of the fistula $[9,11]$.

At the onset of sepsis, broad spectrum antibiotics should be initiated, and cultures should be sent from all possible sources of infection. Antibiotics should then be tailored to culture data, and reserved for patients with evidence of ongoing sepsis, with special consideration given to cases with fungal infections [11]. The indiscriminate use of antibiotics in patients with ECF leads to the selection of highly resistant pathogens that can lead to untreatable overwhelming infections, and should be avoided.

\section{Electrolytes and Nutrition}

Once sepsis has been controlled, the nutritional needs of the patient should be addressed. This patient population is tremendously catabolic. The traditional starting point has been the Harris-Benedict equation, but more recently, the use of indirect calorimetry has allowed the assessment of respiratory quotient and macronutrient balance [11, 27]. The use of parenteral nutrition has classically been a well- 
established part of the management of ECF [18]. Whether used independently or in conjunction with enteral feeding, institution of parenteral nutrition can help meet the nutritional demands of the patient while allowing for minimization of enteric flow through the fistula. Once baseline needs have been calculated, ongoing clinical assessment with appropriate adjustment based on clinical course is crucial. In general, patients with ECF require 25-32 kcal/ $\mathrm{Kg} / \mathrm{day}$, with a calorie to nitrogen ratio ranging from 150 to $200: 1$, and a protein intake of $1.5 \mathrm{~g} / \mathrm{Kg} /$ day, taking into account adjustments for metabolic stress and fistula losses $[11,27]$. Overfeeding should be avoided in order to minimize hyperglycemia, fluid retention, hypercarbia, lipogenesis, and hepatic cholestasis.

While the reduction of flow through the enteric tract (and thus, fistula output) can be associated to increased non-operative closure rates, enteral nutrition is an important factor in preventing sepsis by improving hepatic protein synthesis, maintaining the mucosal barrier integrity, and optimizing the immune and hormonal function of the bowel $[11,28]$. This can be accomplished with as little as $20 \%$ of calories administered enterally. Low output fistulas may allow oral or gastric enteral supplementation, while "feeding through the fistula" is possible if the fistula is proximal enough to allow at least five feet of small bowel absorption to occur in the absence of distal obstruction. It should be kept in mind that the goal of enteral nutrition in patients with ECF is to promote nutritional optimization and mucosal immunity, not fistula closure.

The use of enteral or parenteral glutamine supplementation in critically ill surgical patients has been studied and literature has shown a reduction in infection rates, but not mortality $[27,29,30]$.

Close attention should be paid to levels of vitamin and trace minerals, as these are often deficient in the setting of fistula losses [11]. Some authors advocate for the use of twice the daily allowance of trace minerals, Zinc and vitamin $C$ [11, 27, 31]. Malnutrition has been associated with higher risk of recurrence and mortality with both operative and non-operative management $[3 \bullet, 7,9,26]$.

\section{Output Control, Wound Care, and Skin Protection}

Containment of fistula output and skin protection should be instituted as soon as the diagnosis is made as it will decrease local skin excoriation and inflammation, pain and infection. A skilled wound and ostomy nurse contributes greatly to the care of the patient. While low output fistulas may be controlled with a simple absorbent dressing, complex fistulas often require advanced techniques including barrier creams, powders, and sealants to protect the skin from auto-digestion as well as bridging for fistula

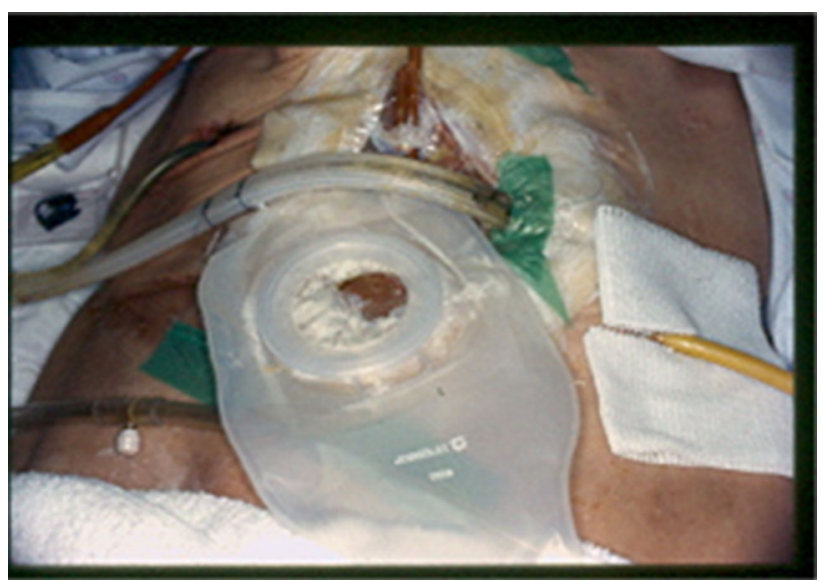

Fig. 1 Control of fistula drainage and skin protection using advanced pouching techniques: sump drainage of the abscess cavity and fistula, wound bridging and isolation of stomatized fistula and wide skin protection with customized skin barriers and sealants

isolation, topographical enhancements, and complex pouching systems with or without sump drainage (Fig. 1).

Improved perioperative care and increasing use of damage control surgical techniques have resulted in an increasing number of patients with EAF, comprising as much as $25 \%$ of cases in recent series [19]. The incidence of EAF is thought to be $5-19 \%$ in patients who have undergone damage control laparotomy and survived long enough to develop complications [25, 27, 32-34]. These patients pose a unique set of problems with skin protection, fluid loss, and involvement of surrounding bowel in the setting of pre-existing critical illness. (Fig. 2).

Negative pressure dressings have been a relatively recent development in the management of complex wounds. They have the advantage of being able to protect the skin in patients with complex fistulas where simpler devices do not suffice [2, 21, 35] (Figs. 3, 4). While several authors have published small series on the use of VAC devices to protect the skin, promote contracture of the wound, or close the fistula [2, 27, 36-38], concern has arisen that these devices can actually bring about the development of recurrent ECF with increased associated mortality $[6,39,40]$. Additionally, in a series scrutinizing the use of VAC devices as temporary abdominal closures in patients undergoing damage control laparotomy, Bee et al. found that, although not statistically significant, fistula formation was greater in the group managed with VAC than with polyglactin mesh [41]. Given the concern that negative pressure therapy can cause bowel injury and lead to fistula formation, the authors advise caution with its use, and recommend the interposition of a layer of material between the bowel and the sponge of the device when it is used. 


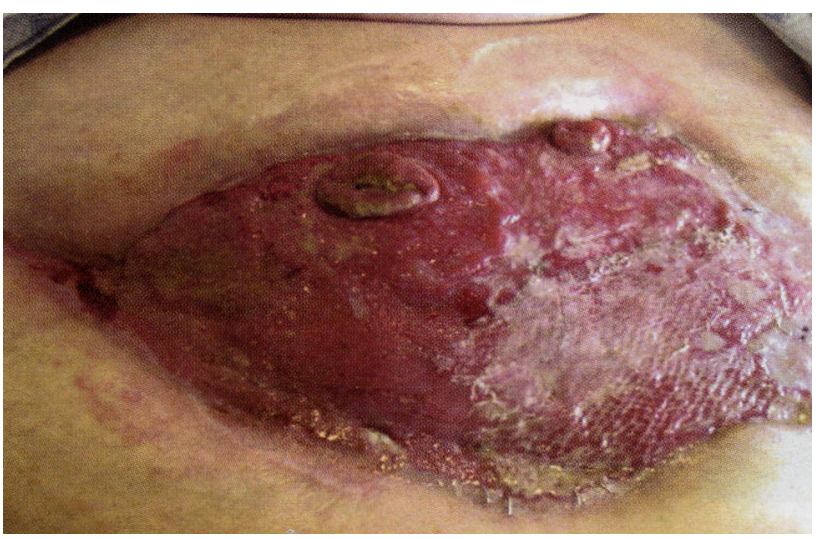

Fig. 2 EAF note 2 stomatized fistula buds and large area of granulation tissue with partially successful split thickness skin graft

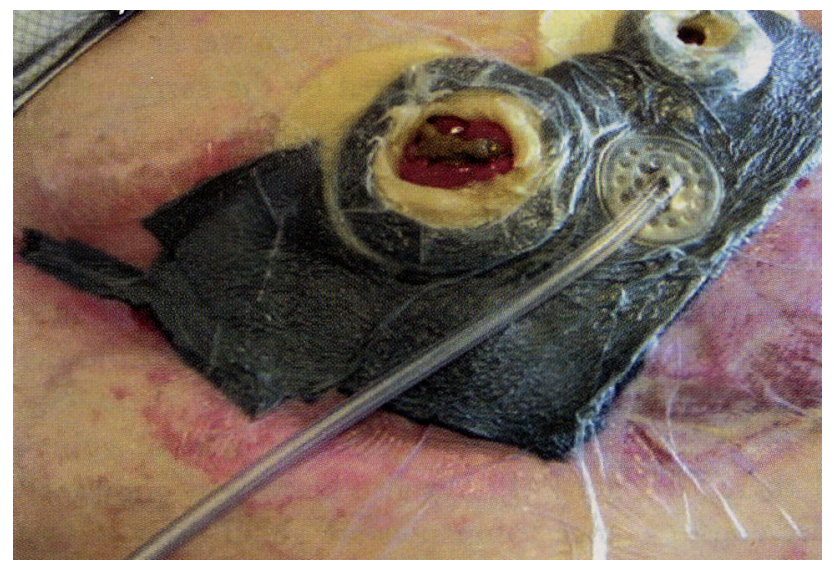

Fig. 3 Two isolated fistula buds surrounded by negative pressure wound dressing

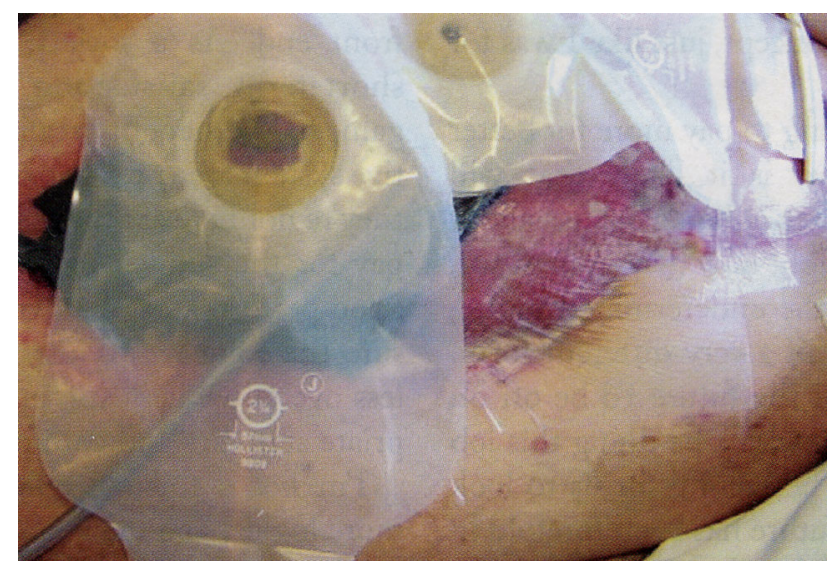

Fig. 4 Final pouching of isolated fistula buds

Whatever containment system is utilized, it must allow for patient mobility and comfort. It should be odor proof, relatively inconspicuous and, if possible, capable of being applied and repaired by general nursing staff, the patient, or their family. A system that can stay in place for at least $72 \mathrm{~h}$ and is amenable to revision or repair is highly desirable. A well-maintained containment system can ameliorate some of the inevitable psychological and social consequences that affect these patients.

Somatostatin and its long-acting analog Octreotide have been studied for their role in decreasing fistula effluent. The literature confirms that they are associated with decreased output and a decreased time to closure in highly favorable fistulas that are most likely destined to close without surgery [2, 11, 42], but not necessarily increased non-operative closure rate [43-46]. Some studies document utility in higher output fistulas to help minimize fluid and electrolyte loss and protect the skin. Authors of these studies recommend trial of Octreotide in selected patients and continuation of therapy if there is a significant reduction in fistula output within 3 days [2]. Problems associated with the use of Octreotide include possible adverse effect on immune function [47] as well as decreased perfusion in the splanchnic and portal circulation, worsening cholestasis [48], not to mention cost.

There has been interest in the use of Infliximab in patients with ECF due to reported success with treatment of perianal fistulas in patients with IBD $[49,50]$. The role remains to be defined as these studies were small and nonrandomized. The utility of this treatment strategy in typical ECF patients will be limited by the fact that it should not be used in the setting of ongoing infections [11]. Acid suppression with H2-blockers and proton pump inhibitors may aid with the prevention of stress ulcers and decrease the fistula output, but has not been shown to increase closure rates. [11].

The use of fibrin glue to seal low output fistulas has been reported in small series, sometimes used in serial injections at the surgeon's discretion [2,51-53]. While results have been largely disappointing, there seems to be some utility in carefully selected patients with favorable fistula anatomy and physiology [2]. It should be noted that this treatment modality carries attendant risk of allergic reaction, prion transmission and air embolism if injected under pressure [43].

Biologic "fistula plugs" have been utilized with some success in ano-rectal fistulas. There is little evidence that these pre-made plugs are of any value in plugging and closing enterocutaneous fistulas. The authors have some experience with customized biologic fistula plugs that are tailored to each case based on 3-dimensional radiographic analysis of the fistula tract. The results have been disappointing thus far.

\section{Radiographic Investigation}

Once the patient is stabilized, radiographic investigation of the fistula to define its anatomy and characteristics can be 
accomplished. A well-performed water-soluble "fistulogram" via small catheters intubating all fistula orifices by a senior radiologist with the attending surgeon present can provide a wealth of information. It will define the source of the fistula, the length and course of its tract, the presence or absence of bowel continuity, inflamed or strictured bowel, the presence of distal obstruction and characteristics of associated abscess cavities. Water-soluble contrast enemas through the rectum and any ostomy will rule out gross colonic or distal small bowel obstruction not seen on other studies. CT scans, can also visualize abscess cavities, define the local environment surrounding the fistula, and show abdominal wall defects as well as the status of the abdominal wall musculature. MRI is valuable in patients whose fistulas are due to malignancy or Crohn's disease and helps to define pre-existing shortened intestines. Foregut studies of the gastrointestinal tract play a role in the evaluation of fistulas associated with the esophagus, stomach, or duodenum.

\section{Definitive Management}

Following investigations, it can become clear for which fistulas have characteristics favorable for spontaneous closure. Nevertheless, a trial of non-operative therapy lasting at least 4 weeks is employed in almost all patients. This trial consists of zero or minimal oral intake, total parenteral nutrition (with or without Octreotide), and mechanical wound care. Patients with very distal or low output fistulas may be allowed to eat or at least take nutritional supplements. Ideally, the measures described above should allow for fistula closure in approximately 4-6 weeks. If successful, diet is advanced and nutritional support is weaned slowly then discontinued when the patient consumes at least $70 \%$ of their protein and caloric requirements. $90 \%$ of fistulas destined to close spontaneously will do so in within this period [7, 43]. In reality, this only occurs in approximately $30 \%$ of fistulas [8, 54•, 55]. If after 4 weeks of non-operative management, decreased output and closure of the fistula is not noted, or if the fistula recurs with the resumption of oral intake, the non-operative trial is considered a failure and planning for operative intervention should be initiated [11]. The decision to proceed with operative intervention requires consideration of the likelihood of closure based on fistula characteristics, physiological and nutritional status of the patient, technical feasibility, and time since the last operation [3•, 11, 24].

Timing of surgery is critical. Intervention before the resolution of sepsis, obliterative peritonitis, or malnutrition is doomed to fail and may prove fatal. Obliterative peritonitis is the development of dense vascular adhesions that form as a result of intra-abdominal inflammation $[55,56$, 57]. In the normal post-operative patient, this is thought to resolve after approximately 6 weeks, but in a fistula patient can take up to 12 months [24]. A soft, pliable abdomen, rather than a firm, "woody" abdomen, and free movement of the surgeon's finger in all directions with digitalization of a stoma or fistula tract suggests an easier abdomen to enter and dissect. Lynch et al. [58] advocate a waiting a period of 12-36 months before attempting operative repair. In contrast, the authors' experience with such prolonged delays is that the increased risk of central line-associated blood stream infections and cholestatic liver injury, among other issues, counteracts the benefit. In a series from the author's own institution, postponing surgical correction for greater than 1 year increased the risk of recurrent fistula following operative intervention twofold [3•]. Other series have also found increased recurrence rates with prolonged waits between diagnosis and operation [5]. Many experts advocate waiting a period of 4-6 months from the time the fistula was identified, and this is the practice to which the authors adhere $[3 \cdot, 11]$. Once a potential date for surgery is identified, continued nutrition support and rehabilitation in the home setting are best. Enteral nutrition by mouth and/or by feeding the fistula is attempted, but can be limited by increased fistula output in the former and mechanical complications in the latter. Complex pouching systems, particularly those involving suction, can be almost impossible in the home setting and often mandate inpatient stay at a hospital or long-term care facility until the time for definitive closure arrives.

\section{Definitive Surgical Closure}

The goal of fistula surgery is re-establishment of GI continuity and soft tissue coverage of intra-abdominal contents with abdominal wall closure. These operations are usually of a prolonged, grueling nature requiring patience and meticulous technique. The patient, their family, and surgical team should prepare accordingly. A thorough discussion should be held with the patient and their family regarding the procedure, its length, expected outcomes, potential complications (including recurrence) and the prolonged nature of post-operative recovery. The case should be discussed with anesthesia and an ICU bed reserved. Bowel preparation should be performed, blood products reserved, and appropriate antibiotic prophylaxis administered.

\section{In General, Steps are the Following}

Incision should be made through "virgin tissue" or over the liver, if possible. This is done to minimize the risk of injuring loops of bowel adherent to the abdominal wall up on entering the abdomen. The incision should be planned 
taking into account the defect associated with the fistula and the need for abdominal wall reconstruction (Figs. 5, 6).

Extensive, meticulous lysis of adhesions then ensues. All adhesions to the abdominal wall and inter-loop adhesions from the ligament of Treitz to the rectum should be lysed. This portion of the operation by itself can often take several hours and must be performed with great care. Any enterotomies or serosal injuries should be carefully and painstakingly repaired.

Once the entirety of the bowel has been mobilized, the next step is isolation of the "fistula island" and resection of the fistula bearing segment of intestines, followed by primary anastomosis (Figs. 7, 8). While the anastomosis can be either hand sewn in 2 layers or stapled, it is the authors' opinion that in these unique cases, hand sewn anastomoses are less likely to leak or bleed post-operatively. One study

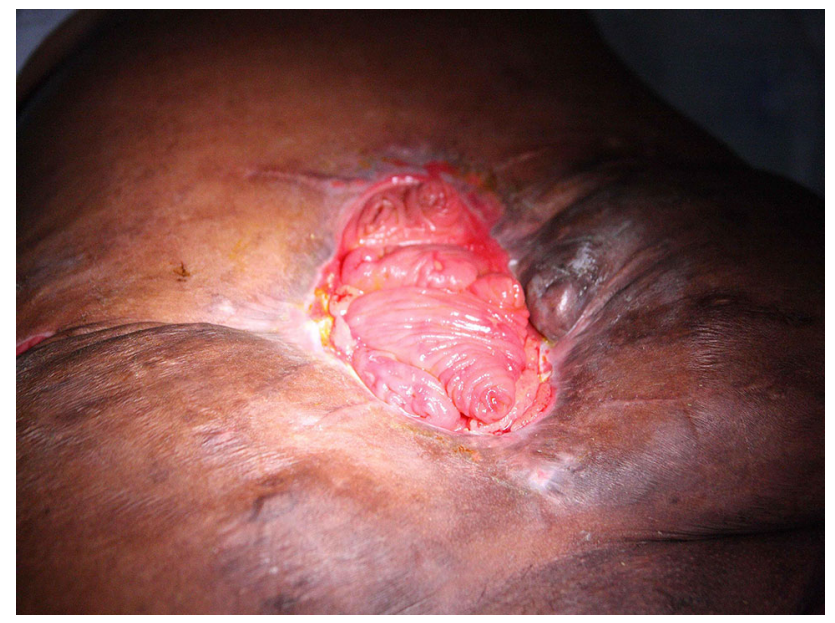

Fig. 5 Multiple stomatized fistula buds in complex enteroatmospheric fistula with large abdominal wall defect

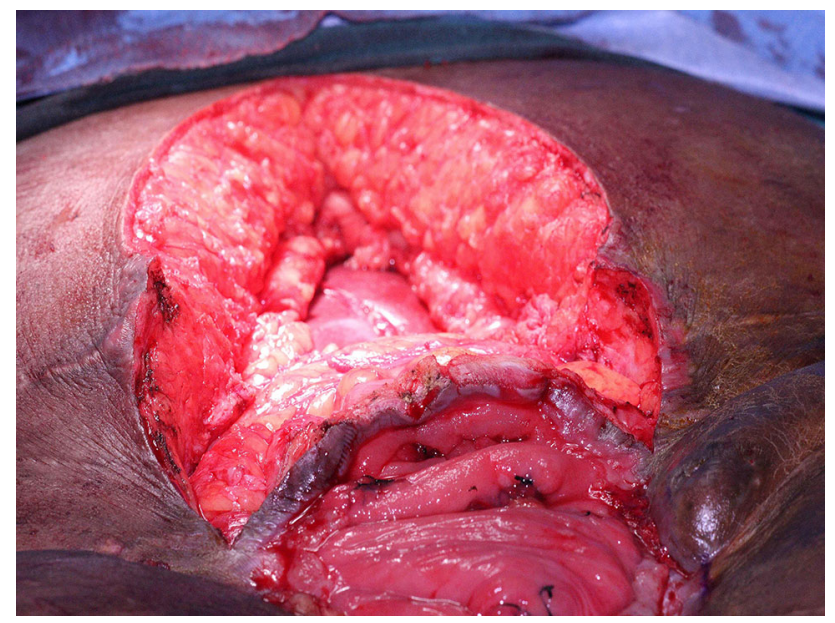

Fig. 6 Enerting the abdominal cavity through virgin territory with an incision above the EAF bed. Note the liver seen at the base of the apical portion of the wound

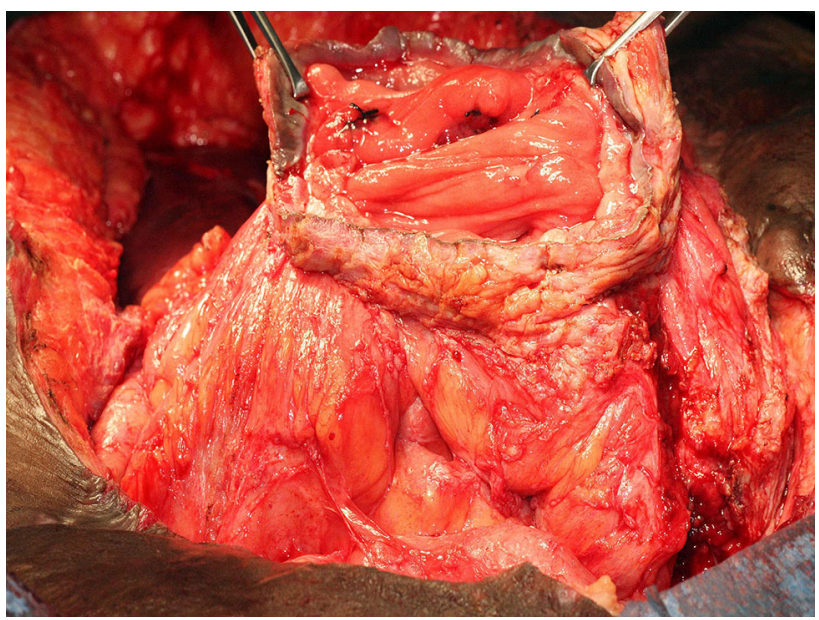

Fig. 7 Creation of the "Fistula Island"

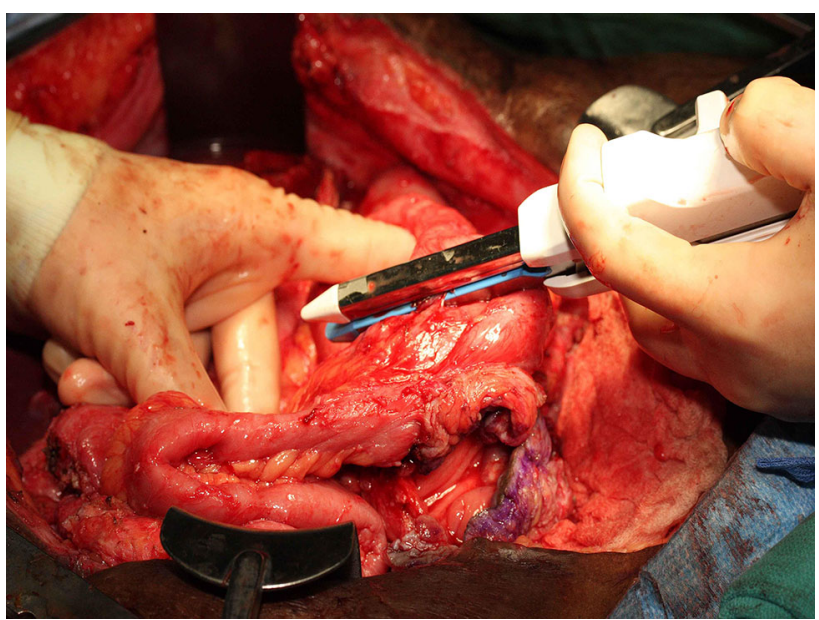

Fig. 8 Resection of the fistula bearing segments of small bowel

confirms this opinion [5], and it is also supported in discussion with other experts in the field. Many series have demonstrated higher recurrence rates when surgical correction involves over-sewing the fistula versus resection with primary anastomosis $[4,18,54 \cdot]$. Staged approaches involving resection of the fistula bearing bowel without anastomosis or proximal diversion of the anastomosis have been advocated by some to decrease recurrence of the fistula but expose the patient to the risk of additional surgery [59].

Abdominal wall closure and soft tissue coverage is paramount to the success of the endeavor, and must be planned carefully $[3 \cdot, 11,24,60]$. It is common to have very large muscular defects once the "fistula island" is resected, making primary fascial closure impossible. The use of permanent mesh is discouraged in light of the fact 
that the operative field following fistula takedown is contaminated by definition, and its placement leads to infection and re-fistulization [60]. A variety of options exist to mobilize the fascial layers of the abdominal wall to bridge the gap, such as component separation or an Abramson's closure, but surgeons are frequently faced with defects too large to cover with these techniques [60]. Recently, the availability of biologic meshes seemed a promising answer to this problem. These meshes were touted as being more resistant to infection, thus amenable to use in an infected field. Some series have emerged suggesting that the use of these meshes alone can lead to infection and re-fistulization, whereas the use of polyglactin mesh was associated to a lower rate of re-fistulization, but with an expected late incisional hernia [12, 61].

The authors of this paper use a composite reconstruction consisting of a 4-ply layer of polyglactin mesh inlay over the bowel as a "neo-omentum", followed by a porcine biologic mesh "blowout" patch, with primary approximation of the muscles over the mesh if possible. Muscular approximation is facilitated by the use of component separation if feasible. The subcutaneous and sub-fascial spaces are widely drained. This reconstruction can be accomplished in most cases but is not without significant problems. Wound complication rates range from 25 to $50 \%$ and can be even higher in obese patients. Early incisional hernia rates are 20-30\% and further loss of abdominal integrity occurs over time given thinned out musculature after component separation and stretching of the biologic mesh with poor collagen formation. Parastomal hernia is common if an ostomy is required.

Some situations of recurrent or recalcitrant fistulas call for additional soft tissue coverage. In institutions that have plastic and reconstructive surgery services available, a multidisciplinary approach to abdominal wall reconstruction can be taken to mobilize healthy tissue from other areas of the body and cover suture lines with healthy, wellperfused tissue [54•]. Pedicled flaps, such as the lateral thigh flap based on the lateral circumflex femoral artery or a Latissimus Dorsii flap, can be utilized in combination with component separation and biologic mesh to accomplish closure of extremely large abdominal wall defects and yield reasonable cosmetic results.

\section{Post-Operative Recovery}

As with any operation, meticulous and comprehensive care optimizes outcome. Most of these patients should be placed in the ICU for hemodynamic and pulmonary monitoring. The authors have, in approximately $25 \%$ of cases, seen systemic inflammatory response syndrome in the early post-operative period, particularly after a long, tedious dissection. Aggressive early goal directed therapy to maintain maximal oxygen delivery can decrease the incidence of multiple organ dysfunction and mortality. Continued nutrition support ensures adequate nitrogen and calories for healing of the anastomosis and abdominal wall closure. Post-operative ileus is often prolonged, so TPN is essential until the patient can consume orally or tolerate via tube feedings $75 \%$ of calculated energy requirements. High-dose vitamin $\mathrm{C}$ and Zinc supplementation are continued to promote healing. Early trophic enteral feedings before adequate bowel sounds or passage of stool and flatus are not given in this unique population of patients. When enteral tube feedings are utilized, the use of elemental or semi-elemental dipeptide formulas is advised since, in some cases, the intestines may be atrophic from disuse. Oral glutamine supplementation may enhance restoration of gut mucosal integrity. Significant post-operative anemia can impair anastomotic and abdominal wall healing. Finally, post-operative systemic antibiotics are continued for $72 \mathrm{~h}$ in simple fistula closures where contamination is minor, about up to 10 days in more complex situations where contamination is greater. There is, of course, no science behind this except for the knowledge that these patients are often immunosuppressed and that post-operative sepsis of any type increases fistula recurrence rates.

\section{Outcomes}

As previously noted, overall mortality for enterocutaneous fistulas ranges from 10 to $30 \%$. Medical management of ECF leads to spontaneous closure in approximately $30 \%$ of patients in most series. Most patients ultimately require definitive surgical closure which carries a 30 day operative mortality rate of 3-5\% and a 1 year mortality rate of 7-19\% from fistula-related complications. The series with higher mortality rates routinely have larger sub-populations of patients with more complex fistulas. Operative success in closing the fistula and keeping it closed is 75-89\%. Specifically, after definitive surgery, simple fistulas recur only $5 \%$ of the time, while complex fistulas recur up to $30 \%$ of the time in most series. Factors besides complexity associated with recurrent fistulization after definitive surgical closure vary among series. Etiological factors, such as IBD, malignancy, radiation, as well trauma with subsequent enteroatmospheric fistula development, are associated with increased recurrence [5, 62, 63•]. Other risk factors for recurrence also include pre-op comorbidities, such as liver disease, portal hypertension, cardiopulmonary disease, chronic renal failure, steroid use, and pre-op short bowel syndrome, as well as post-operative complications, such as respiratory failure, organ space surgical site infections, and anemia requiring transfusion $[3 \bullet, 63 \bullet$. Noncolonic fistulas, high output fistulas, and fistulas treated with non-resective techniques were also more likely to recur $[5,19]$. 


\section{Conclusion}

Enterocutaneous fistulas remain one of the most challenging problems in surgery. Unfortunately, the incidence appears to be rising as surgeons attempt increasingly complex operations in older patients with higher acuity and multiple comorbidities. A dedicated, multidisciplinary approach is paramount in restoring gastrointestinal tract continuity while limiting morbidity, mortality, and fistula recurrence. Referral to tertiary centers that have developed the necessary components and expertise to ensure maximal patient outcomes is, of course, advised.

\section{Compliance with Ethics Guidelines}

Conflict of Interest Carla I. Haack, John R. Galloway, and Jahnavi Srinivasan declare no conflicts of interest.

Human and Animal Rights and Informed Consent This article does not contain any studies with human or animal subjects performed by any of the authors.

\section{References}

Papers of particular interest, published recently, have been highlighted as:

- Of importance

1. Joyce M, et al. Management of complex gastrointestinal fistula. Curr Probl Surg. 2009;46(5):384-430.

2. Draus J, et al. Enterocutaneous fistula: are treatments improving? Surgery. 2006;140(4):570-8.

3. - Owen, R. et al. (2013), Definitive surgical treatment of enterocutaneous fistula. JAMA, 48(2), 118-126. Recent large series with a high concentration of patients with more complex fistulas(category 3, and 4 as well as Enteroatmospheric).

4. Lynch J, et al. Clinical outcome and factors predictive of recurrence after enterocutaneous fistula surgery. Ann Surg. 2004;240(5): 825-31.

5. Brenner M, et al. Risk factors for recurrence after repair of enterocutaneous fistula. Arch Surg. 2009;144(6):500-6.

6. Lundy J, et al. Historical perspectives in the care of patients with enterocutaneous fistula. Clin Colon Rectal Surg. 2010;23(3): $133-41$.

7. Ruben G. Treatment strategies in 135 consecutive patients with enterocutaneous fistulas. World J Surg. 2008;32:445-53.

8. Murphy $\mathrm{J}$, et al. Establishing a regional enterocutaneous fistula service: the royal London hospital experience. Int J Surg. 2013;11(9):952-6.

9. Hollington $\mathrm{P}$, et al. An 11 year experience of enterocutaneous fistula. Br J Surg. 2004;91:1646-51.

10. Datta V, et al. The management of enterocutaneous fistula ina regional unit in the United Kingdom: a prospective study. Dis Colon Rectum. 2010;53(2):192-9.

11. Evenson A, et al. Current management of enterocutaneous fistula. J Gastrointest Surg. 2006;10(3):455-64.

12. Connolly $\mathrm{P}$, et al. Outcome of reconstructive surgery for intestinal fistula in the open abdomen. Ann Surg. 2008;247:440-4.
13. Edmunds L, et al. External fistulas arising from the gastrointestinal tract. Ann Surg. 1960;152:445-71.

14. Chapman R, et al. Management of intestinal fistulas. Am J Surg. 1964;108:157-64.

15. Berry S, et al. Classification and pathophysiology of enterocutaneous fistulas. Surg Clin North Am. 1996;76:1009-18.

16. Campos A, et al. A multivariate model to determine prognostic factors in gastrointestinal fistulas. J Am Coll Surg. 1999;188:483-90.

17. Levy E, et al. High-output external fistulae of the small bowel: Management with continuous enteral nutrition. Br J Surg. 1989;76:676-9.

18. Soeters P, et al. Review of 404 patients with gastrointestinal fistulas: impact of parenteral nutrition. Ann Surg. 1979;190:189-202.

19. Martinez J, et al. Systematic management of postoperative enterocutaneous fistulas: factors related to outcomes. World J Surg. 2008;32:436-43.

20. Harriman $\mathrm{S}$, et al. The C-reactive protein-to-prealbumin ratio predicts fistula closure. Am J Surg. 2011;202(2):175-8.

21. Dionigi G, et al. Treatment of high output entero-cutaneous fistulae associated with large abdominal wall defects: single center experience. Int J Surg. 2008;6:51-6.

22. Fischer $\mathrm{J}$, et al. The pathophysiology of enterocutaneous fistulas. World J Surg. 1983;7(4):446-50.

23. Bradley $\mathbf{M}$, et al. Independent predictors of enteric fistula and abdominal sepsis after damage control laparotomy. JAMA Surg. 2013;148(10):947-57.

24. Schecter W, et al. Enteric fistulas: principles of management. J Am Coll Surg. 2009;209(4):484-91.

25. Dubose J, et al. Enterocutaneous fistulas in the setting of trauma and critical illness. Clin Colon Rectal Surg. 2010;23(3):182-9.

26. Mawdsley J, et al. An analysis of predictive factors for healing and mortality in patients with enterocutaneous fistulas. Aliment Pharmacol Ther. 2008;28(9):1111-21.

27. Majercik $\mathrm{S}$, et al. Enteroatmospheric fistula: from soup to nuts. Nutr Clin Pract. 2012;27:507-12.

28. Gianotti L, et al. Role of early enteral feeding and acute starvation on postburn bacterial translocation and host defense: prospective, randomized trials. Crit Care Med. 1994;22:265-72.

29. Aguilar-Nacimiento De, et al. Oral glutamine in addition to parenteral nutrition improves mortality and the healing of highoutput intestinal fistulas. Nutr Hosp. 2007;22(6):672-6.

30. Yue $\mathrm{C}$, et al. The impact of perioperative glutamine-supplemented parenteral nutrition on outcomes of patients undergoing abdominal surgery: a meta-analysis of randomized clinical trials. Am J Surg. 2013;79(5):506-13.

31. Dudrick $\mathrm{S}$, et al. Artificial nutritional support in patients with gastrointestinal fistulas. World J Surg. 1999;23:570-6.

32. Miller R, et al. Complications after 344 damage-control open celiotomies. J Trauma. 2005;59(6):1365-74.

33. Jamshidi R, et al. Biological dressings for the management of enteric fistulas in the open abdomen: a preliminary report. Arch Surg. 2007;142(8):793-6.

34. Tsuei B, et al. The open peritoneal cavity: etiology correlates with the likelihood of fascial closure. Am J Surg. 2004;70(7): $652-6$.

35. Al-Khoury G, et al. Improved control of exposed fistula in the open abdomen. J Am Coll Surg. 2008;206(8):397-8.

36. Cro $\mathrm{C}$, et al. Vacuum assisted closure system in the management of enterocutaneous fistulae. Postgrad Med. 2002;78:364-5.

37. Alvarez A, et al. Vacuum-assisted closure for cutaneous gastrointestinal fistula management. Gynecol Oncol. 2001;80(3):413-6.

38. Erdmann D, et al. Abdominal wall defect and enterocutaneous fistula treatment with the vacuum assisted closure system. Plast Reconstr Surg. 2001;108:2066-8.

39. Fischer J. A cautionary note: the use of vacuum-assisted closure systems in the treatment of gastrointestinal cutaneous fistula may 
be associated with higher mortality from subsequent fistula development. Am J Surg. 2008;196:1-2.

40. Rao M, et al. The use of vacuum assisted closure of abdominal wounds: a word of caution. Colorectal Dis. 2007;9(3):266-8.

41. Bee $\mathrm{T}$, et al. Temporary abdominal closure techniques; a prospective randomized trial comparing polyglactin 910 mesh and vacuum- assisted closure. J Trauma. 2008;65(2):337-44.

42. Nubiola $P$, et al. Treatment of 27 post-operative enterocutaneous fistulas with the long half-life somatostatin analogue SMS 201-995. Ann Surg. 1989;210:56-8.

43. Galie K, et al. Postoperative enterocutaneous fistula: when to reoperate and how to succeed. Clin Colon Rectal Surg. 2006; 19(4):237-46.

44. Torres A, et al. Somatostatin in the management of gastrointestinal fistulas. A multicenter trial. Arch Surg. 1992;127:97-9.

45. Sancho J, et al. Randomized double blind placebo-controlled trial of early octreotide in patients with postoperative enterocutanous fistula. Br J Surg. 1995;82:638-41.

46. Hesse U, et al. Role of somatostatin-14 and its analogues in the management of gastrointestinal fistulae: clinical data. Gut. 2002;49(SupplIV):iv1-20.

47. Lattuada D, et al. Inhibitory effect of pasireotide and octreotide on lymphocyte activation. J Neuroimmunol. 2007;182:153-9.

48. Cooper A, et al. Fasting and post-prandial splanchnic blood flow is reduced by a somatostatin analogue (octreotide) in man. Clin Sci. 1991;81:169-75.

49. Present D, et al. Infliximab for the treatment of patients with Crohn's disease. N Engl J Med. 1999;340:1398-405.

50. Viscido A, et al. Infliximab in refractory pouchitis complicated by fistulae following ileo-anal pouch for ulcerative colitis. Aliment Pharmacol Ther. 2003;17:1263-71.

51. Rabago L, et al. Endoscopic treatment of postoperative fistulas resistant to conservative management using biological fibrin glue. Endoscopy. 2002;34:632-8.
52. Truong S, et al. Results after endoscopic treatment of postoperative upper gastrointestinal fistulas and leaks using combined Vicryl plug and fibrin glue. Surg Endosc. 2004;18:1105-8.

53. Lamont $\mathrm{J}$, et al. Closure of proximal colorectal fistulas using fibrin sealant. Am J Surg. 2002;68:615-8.

54. Martinez, J. et al. (2012) Factors predictive of recurrence and mortality after surgical repair of enterocutaneous fistula. J Gastrointest Surg, 16, 156-164. Recent study defining the factors influencing outcomes in this patient population.

55. Cohen M, et al. The use of muscle flaps for the management of recalcitrant gastrointestinal fistula. Clin Plast Surg. 2006;33: 295-302.

56. Hill G, et al. Operative strategy in the treatment of enterocutaneous fistulas. World J Surg. 1983;7:495-501.

57. Fazio V, et al. Factors influencing the outcome of treatment of small bowel cutaneous fistula. World J Surg. 1983;7:481-8.

58. Lynch A, et al. Clinical outcome and factors predictive of recurrence after enterocutaneous fistula surgery. Ann Surg. 2004;240(5):825-31.

59. Runstrom B, et al. Outcome of 132 consecutive reconstructive operations for intestinal fistula- staged operation without primary anastomosis improved outcome in retrospective analysis. Scand J Surgery. 2013;102:152-7.

60. Fischer $J$, et al. The importance of reconstruction of the abdominal wall after gastrointestinal fistula closure. Am J Surg. 2009;197:131-2.

61. Carbonell A, et al. The susceptibility of prosthetic biomaterials to infection. Surg Endosc. 2005;19:430-5.

62. Redden M, et al. The etiology of enterocutaneous fistula predicts outcome. Ochsner J. 2013;13(4):507-11.

63. •Rahbour, G. et al. (2013) Seven-year experience of enterocutanous fistula with univariate and multivariate analysis of factors associated with healing: development of a validated scoring system. Colorectal Dis, 15, 1162-1170. First study that has led to a scoring system to predict fistula healing. 